\title{
Identification of Novel Genes Associated with Litter size of indigenous Sheep Population in Xinjiang, China using Specific- Locus Amplified Fragment Sequencing Technology
}

\author{
Haiyu Ma ${ }^{1}$, Chao Fang ${ }^{2}$, Lingling Liu ${ }^{1}$, Qiong Wang ${ }^{1}$, Jueken Aniwashi ${ }^{1}$, Yiming Sulaiman ${ }^{1}$, Kezierkailedi \\ Abudilaheman ${ }^{3}$, Wujun Liu ${ }^{\text {Corresp. } 1}$ \\ ${ }^{1}$ College of Animal Science, Xinjiang Agriculture University, Urumqi, Xinjiang, China \\ 2 Department of veterinary managment of animal resources,Faculty of Veterinary Medicine, University of Liège, Liège, Belgium \\ 3 People's Congress of Xinjiang Uygur Autonomous Region, Urumqi, Xinjiang, China \\ Corresponding Author: Wujun Liu \\ Email address: Iwj_ws@163.com
}

Background. Xinjiang in China has a diverse ecological system with abundant sheep breed resources, which include several high-litter size sheep populations. Previous studies have confirmed that the major high prolificacy gene cannot be used to detect high litter size. Our research team found a resource group in Pishan County, southern Xinjiang, with a multi-breast rate $>60 \%$. It showed high fertility with an average litter size of two to four in one birth, excellent breast development, and a high survival rate of lambs. In the present study, we used this resource as an ideal sample for studying the genetic mechanisms of high prolificacy in sheep.

Methods. Indigenous sheep populations from Xinjiang, with different litter size, were selected for the research, and specific-locus amplified fragment sequencing (SLAF-seq) technology was used to comprehensively screen single nucleotide polymorphisms (SNPs) from the whole genome that may cause differences in litter size. Novel genes associated with litter size of sheep were detected using genomewide association studies (GWAS), providing new clues revealing the regulation mechanism of sheep fecundity. Candidate genes related to ovulation and litter size were selected for verification using Kompetitive Allele Specific polymerase chain reaction (KASP) cluster analysis.

Results. We identified 685,300 SNPs using SLAF-seq technique for subsequent genome-wide analysis. Subsequently, 155 SNPs were detected at the genome-wide level. Fourteen genes related to sheep reproduction were notated: COIL, SLK, FSHR, PIXna3, Ddx24, CXCL12, Pla2g7, ATP5F1A, KERA, GUCY1A1, LOC101107541, LOC101107119, LOC101107809, and BRAF. Based on literature reports, 30 loci of seven genes and candidate genes (CXCL12, FSHR, SLK, GUCY1A1, COIL, LOC101107541, and LOC101107119) related to ovulation and litter size were selected for verification using KASP cluster analysis. Among them, nine loci of three genes were successfully genotyped. Three loci of FSHR (GenBank ID: 443299, g. 75320741G >A site), GUCY1A1 (GenBank ID: 101110000, g. 43266624C>T site), and COIL (GenBank ID: 101123134, g. $7321466 \mathrm{C}>\mathrm{G}$ site) were found to be significantly or extremely significantly associated with litter size. These three loci are expected to be used as molecular markers to determine differences in litter size in sheep. 


\section{Identification of Novel Genes Associated with Litter size of}

\section{2 indigenous Sheep Population in Xinjiang, China using}

3 Specific-Locus Amplified Fragment Sequencing Technology

4

5

Haiyu $\mathrm{Ma}^{1}$, Chao Fang ${ }^{2}$, Lingling Liu ${ }^{1}$, Qiong Wang ${ }^{1}$, Jueken Aniwashi ${ }^{1}$, Yiming Sulaiman ${ }^{1}$, Kezierkailedi Abudilaheman ${ }^{3}$, Wujun Liu ${ }^{1}$

${ }^{1}$ College of Animal Science, Xinjiang Agricultural University, Urumqi, Xinjiang, China

${ }^{2}$ Department of Veterinary Management of Animal Resources, Faculty of Veterinary Medicine, University of Liège, Liège, Belgium

${ }^{3}$ People's Congress of Xinjiang Uygur Autonomous Region, Urumqi, Xinjiang, China

Corresponding Author:

Wujun Liu ${ }^{1}$

College of Animal Science, Xinjiang Agricultural University, Urumqi, Xinjiang, China Email address: Lwj_ws@163.com

\section{Abstract}

Background. Xinjiang in China has a diverse ecological system with abundant sheep breed resources, which include several high-litter size sheep populations. Previous studies have confirmed that the major high prolificacy gene cannot be used to detect high litter size. Our research team found a resource group in Pishan County, southern Xinjiang, with a multi-breast rate $>60 \%$. It showed high fertility with an average litter size of two to four in one birth, excellent breast development, and a high survival rate of lambs. In the present study, we used this resource as an ideal sample for studying the genetic mechanisms of high prolificacy in sheep.

Methods. Indigenous sheep populations from Xinjiang, with different litter size, were selected for the research, and specific-locus amplified fragment sequencing (SLAF-seq) technology was used to comprehensively screen single nucleotide polymorphisms (SNPs) from the whole genome that may cause differences in litter size. Novel genes associated with litter size of sheep were detected using genome-wide association studies (GWAS), providing new clues revealing the regulation mechanism of sheep fecundity. Candidate genes related to ovulation and litter size 
33 were selected for verification using Kompetitive Allele Specific polymerase chain reaction 34 (KASP) cluster analysis.

35 Results. We identified 685,300 SNPs using SLAF-seq technique for subsequent genome-wide analysis. Subsequently, 155 SNPs were detected at the genome-wide level. Fourteen genes related to sheep reproduction were notated: COIL, SLK, FSHR, Plxna3, Ddx24, CXCL12, Pla2g7, ATP5F1A, KERA, GUCY1A1, LOC101107541, LOC101107119, LOC101107809, and $B R A F$. Based on literature reports, 30 loci of seven genes and candidate genes (CXCL12, FSHR, SLK, GUCY1A1, COIL, LOC101107541, and LOC101107119) related to ovulation and litter size were selected for verification using KASP cluster analysis. Among them, nine loci of three genes were successfully genotyped. Three loci of FSHR (GenBank ID: 443299, g. 75320741G>A site), GUCY1A1 (GenBank ID: 101110000, g. 43266624C>T site), and COIL (GenBank ID: 101123134 , g. $7321466 \mathrm{C}>\mathrm{G}$ site) were found to be significantly or extremely significantly associated with litter size. These three loci are expected to be used as molecular markers to determine differences in litter size in sheep.

\section{Introduction}

Fertility is one of the most important economic traits in sheep. Sheep populations with high reproductive performance show 2 to 3 times higher production efficiency and economic benefit from lambs than those with low reproductive performance do. Therefore, detection of molecular markers of high fecundity in sheep is of great significance in revealing the genetic basis of sheep reproductive traits, improving sheep breeds by molecular breeding, and establishing core groups or breeding new lines. Therefore, reproductive traits have become a research hotspot in sheep breeding.

Among sheep breeds worldwide, few exhibit high litter size, early sexual maturity, and perennial oestrus. Presently, a few major genes affecting high fecundity have been found in Cambridge and Belclare (Hanrahan et al., 2004), Icelandic (Eiriksson et al., 2017), Romanov (Deniskova et al., 2017), Finnish (Mullen \& Hanrahan, 2014), and other high fecundity sheep breeds abroad, whereas domestic studies have mainly focused on Hu (Yue, 1996) and Small Tail Han sheep (He et al., 2012). The results show that different major genes affect the litter size of various sheep breeds. As a result, the major genes identified in small-tailed Han sheep have been selected as markers for domestic mutation detection for sheep litter size, which has led to pseudoscience in this field.

Xinjiang has a diverse ecological system with abundant sheep resources, including several high litter size sheep populations. Previous studies have confirmed the existence of the high 
67 prolificacy major gene $F e c B$ mutation in the Cele black sheep (Jiang et al., 2017) and Duoliang 68 sheep populations (Wang et al., 2017), but it cannot be used to detect high litter size. Our 69 research team found a resource group in Pishan County, southern Xinjiang, for the indigenous 70 prolificacy sheep breed in Xinjiang. The ewes are oestrus all year round mainly because of 71 natural mating and, therefore, they are bred throughout the year with a multi-breast rate of more 72 than $60 \%$. This breed showed high fertility with an average litter size of two to four lambs per 73 birth, excellent breast development, and a high survival rate of lambs. Bashbai sheep are single74 breasted, seasonally oestrus sheep found in the Tacheng area of Xinjiang. Breeding is mainly via artificial insemination, and the oestrus period is typically in November. The lambing rate is $103 \%$, milk yield is stable, and lamb survival rate is $98 \%$. These breeds different considerably in the number of lambs produced. Consequently, we considered them an ideal sample for studying the genetic mechanisms of high prolificacy in sheep.

In this study, ewes with different litter size (one to four) in Xinjiang were selected as research materials. Genome-wide association studies (GWAS) based on the specific-locus amplified fragment sequencing (SLAF-seq) technique were used to identify single nucleotide polymorphisms (SNPs) that might cause differences in litter size.

\section{Materials \& Methods}

\section{Sample Collection and DNA Extraction}

A total of 126 sheep from two populations were used in this study, including 62 Hetian sheep from Pishan county of Hetian city of Xinjiang (East longitude $77^{\circ} 31^{\prime}-79^{\circ} 38^{\prime}$, latitude $35^{\circ} 22^{\prime}-$ $39^{\circ} 01^{\prime}$ ), and 64 Bashbay sheep from Yumin county of Tacheng state of Xinjiang (East longitude $82^{\circ} 12^{\prime}-83^{\circ} 30^{\prime}$, latitude $\left.45^{\circ} 24^{\prime}-46^{\circ} 3^{\prime}\right)$. Whole blood samples $(6 \mathrm{~mL})$ were collected from the jugular veins of the sheep and transferred into ethylenediaminetetraacetic acid (EDTA) anticoagulant tubes. Genomic DNA was extracted using a standard phenol chloroform extraction method (Köchl et al., 2005) for subsequent experiments. The study design was approved by the appropriate ethics review board. The University of Xinjiang Agricultural University approved the use of its facilities for the study (Animal protocol number: 2017010).

\section{Construction of SLAF-seq Library and High-throughput Sequencing}

The current sheep genome (Oar_v4.0) was selected as the reference genome to simulate the restriction enzyme digestion and identify the expected SLAF yield. Hpy166II + EcoRV-HF enzyme was selected for enzymatic digestion (Davey et al., 2013). Furthermore, to evaluate the accuracy of the digestion strategy, Oryza sativa indica (http://rapdb. dna. affrc. go. JP/) was selected (Li et al., 2009b). Subsequently, data of 126 individuals were used to construct the 
101 SLAF library (Kozich et al., 2013) and for sequencing after quality inspection. To evaluate the

102 accuracy of the enzyme test, Nippon Sunshine was selected as the control for sequencing.

103 Analysis of SLAF-seq data and identification of SNP loci

104 According to SLAF tags, the SNP locus information was analysed and screening criteria was

105 set at minor allele frequency $(\mathrm{MAF})>0.05$. The development of SNP markers was based on the

106 sheep reference genome using BWA (Li \& Durbin, 2009) to compare the sequenced reads to the

107 reference genome, and GATK (Mckenna et al., 2010) and SAMtools (Li et al., 2009a) to SNP

108 calling. The intersection of the SNP markers obtained using the two methods was used as the

109 final reliable data set of SNP markers. Sequencing reads of the control were compared with their

110 reference genomes using SOAP software. The double-end contrast efficiency was $92.46 \%$, and

111 the contrast efficiency was normal. The enzymatic cleavage efficiency of the control was

$11293.60 \%$, indicating that the enzymatic cleavage reaction was normal.

\section{Population structure}

114 Principal component analysis (PCA) was performed using PLINK1.9 (Purcell et al., 2007),

115 and the ggplot2 package in R (v3.4.4) was used to generate the PCA figure (Wickham, 2015).

116 We removed the SNPs in linkage disequilibrium in PLINK 1.9 using the command (--indep-

117 pairwise 5050.2 ).

\section{GWAS}

119 Based on SNP analyses, the general linear model (GLM) and mixed linear model (MLM) of the TASSEL (Bradbury et al., 2007) software (http://www.maizegenetics.net) were used to obtain the correlation values. The Q matrix of the sample population structure was calculated using Admixture software (Alexander et al., 2009) and the K matrix of the relationship between samples was calculated using SPAGeDi software (Hardy et al., 2002). The GLM uses the group structure information, whereas the MML uses the information of the population structure and the kinship relationship. The fixed effects of the MLM model are parity and population, the random effects are related, and each SNP site is finally associated with a value. Manhattan chart analysis and Q-Q Plot were used to analyse the population structure and their diagrams were both drawn using R language (Zhiwu et al., 2010).

\section{Bioinformatics analyses}

130 We combined several commonly used bioinformatics databases, such as the National Center

131 for Biotechnology Information (NCBI, http://www.ncbi.nlm.nih.gov/), University of California

132 Santa Cruz (UCSC, http://genome.ucsc.deu/), and Ensembl (http://asia.ensembl.org/index.html)

133 to locate significant SNPs.

134 The position of the significant SNPs was located, and for SNP markers that were not within

135 the gene, candidate genes $500 \mathrm{~kb}$ up-stream and down-stream of the significant SNP site were 
136 searched to determine the linkage disequilibrium between the markers. Candidate gene functions

137 were identified and analysed using the online gene enrichment software DAVID 6.7

138 (https://david.ncifcrf.gov/home.jsp). Venny2.1.0

139 (http://bioinfogp.cnb.csic.es/tools/venny/index.html) was used to draw a Venn diagram of the

140 overlap sites of the two methods.

\section{KASP typing verification}

142 KASP typing was performed at 30 loci of the seven candidate differential genes (FSHR, COIL, 143 GUCY1A1, CXCL12, SLK, LOC101107541, and LOC101107119), which were annotated. Nine

144 pairs of successfully typed primers are listed in Table 1. A one-way analysis of variance

145 (ANOVA) and $t$-test were performed using the statistical package for the social sciences (SPSS)

146 version 19.0 software to analyse the association between litter size and mutant locus genotypes.

147

148

\section{Results}

\section{Establishment of database and sequencing evaluation}

To obtain the actual SLAF tags used in this study, 62 and 64 Hetian and Bashbai sheep, respectively were subjected to SLAF-seq using the same enzyme combinations as those used in computer restriction analysis. A total of $854.88 \mathrm{Mb}$ reads data were obtained for all individuals, and the average Q30 and GC contents were $91.74 \%$ and $42.14 \%$, respectively, indicating that the SLAF-seq process was normal and available. After genome comparison and SNP mining, $5,218,278$ population SNPs were found using all individuals. The completeness was 0.5 times, and the genomic frequency was 0.05 filtered to 685,300 SNPs sites, and 685,300 SNPs were identified for subsequent analysis.

\section{Population stratification assessment}

The results of the PCA (Fig. 1) show that PC1 and PC2 had variances of 3.02\% and 2.15\%, respectively. Furthermore, the results showed that Hetian and Bashbay sheep were separated by $\mathrm{PC} 1$ and there was no mixing between the two populations, which provided a foundation for the subsequent GWAS.

163

\section{GWAS}

164 In this study, GLM and MLM were used to analyse the GWA of litter size traits in Hetian and Bashbai sheep. A Bonferroni correction of $\alpha=0.1 \%$ was applied for genome-wise thresholds (significance threshold $=-\log _{10}[\alpha /$ number of independent SNPs $]$ ). SNPs with p-values below $1.459 \mathrm{e}-07(0.1 / 685300)$ were considered significantly associated with the phenotype. GWAS 
170 traits are shown in Figs. 2 and 3. A total of 25 SNPs were detected using both methods (Fig. 4).

171 The QQ-plot diagram (Figs. 5 and 6) shows a large deviation in the SNP point, which at this site

172 was considered to be caused by the genetic effect of this SNP mutation.

\section{Gene function annotation}

174 Using Oar_v4.0 sequence information of sheep genome and common database information

175 such as that from NCBI, SNP loci with significant GWAS results were analysed and annotated.

176 A total of 133 genes were annotated in the two models, including FRS2, RGS3, MDH1, IMPA1,

177 and $K C N E 3$, which are involved in the differentiation and survival of nerve cells. Some of them

178 are new genes that have not been clearly labelled, and their functions need to be further studied;

17914 of these genes were related to reproduction. COIL, SLK, Plxna3, and Ddx24 genes affect the

180 development of ovaries and follicles in sheep; CXCL12, Pla2g7, ATP5F1A, and KERA affect the

181 early development of the placenta and placental membrane; GUCY1A1, LOC101107541,

182 LOC101107119, and LOC101107809 participate in the oxytocin signalling pathway and

183 indirectly regulate the production of ovarian steroids; and $B R A F$ and $D d x 24$ are the most

184 expressed in the uterus.

\section{Enrichment analysis results}

186 Genecards and DAVID6.7 online websites were used to analyse the functions of the candidate

187 genes while Gene Ontology (GO) and Kyoto Encyclopedia of Genes and Genomes (KEGG)

188 online websites were searched for candidate genes. Enrichment analysis revealed that these

189 differential genes are mainly involved in pathways such as oxytocin signalling, amino acid

190 biosynthesis, neurotrophic signalling, pentose phosphate, and Wnt signalling. In addition to the

191 FSHR, SLK, GUCY1A1, and LOC1001107541 genes, a few others are involved in the oxytocin

192 signalling pathway, which regulates the litter size of sheep by regulating follicle stimulating

193 hormone receptors, and ultimately forms a multi-foetal sheep population.

\section{Functional validation of genes related to litter size regulation}

195 Based on the results of previous studies and comprehensive analysis of gene function, 30

196 missense mutation sites of seven genes (CXCL12, FSHR, SLK, GUCY1A1, Coil,

197 LOC101107541, and LOC101107119) closely related to ovulation and litter size were screened,

198 and 126 individuals with different litter size were typed using KASP. Nine sites of the final three

199 genes (FSHR, GUCY1A1, and COIL) were successfully typed. Only one genotype was identified

200 at 21 loci of the remaining four genes, which could not be used to determine the genotype.

201 Association analysis between different genotypes and litter size

202 The association analysis between successfully typed genotypes and individuals with different

203 litter size was showed significant differences or significant correlations in litter size among 
204 different genotypes of FSHR (g.75320741G>A), GUCY1A1 (g.43266624C > T), and COIL genes

205 (g.7321466C $>$ G, Figs. 7-9).

206 At the g.75320741 locus of the FSHR gene, the average litter size of G/G genotype

207 individuals was significantly higher than that of $\mathrm{A} / \mathrm{A}$ genotype individuals was $(\mathrm{P}=0.004)$. At

208 the g.43266624 locus of the GUCY1A1 gene, the average litter size of C/C genotype individuals

209 was significantly higher than that of $\mathrm{T} / \mathrm{T}$ genotype individuals was $(\mathrm{P}=0.038)$. At g.7321466

210 locus of COIL gene, the average litter size of $\mathrm{C} / \mathrm{C}$ genotype individuals was significantly higher

211 than that of $\mathrm{T} / \mathrm{T}$ genotype individuals $(\mathrm{P}=0.042$, Fig. 10).

\section{Discussion}

\section{Evaluation of reliability of SLAF-seq technology}

214 In this study, the SLAF-seq method used to identify SNPs located in the genome of Chinese 215 indigenous sheep populations detected $>685,300$ SNPs. In addition, 133 genes were annotated

216 by comparing and analysing the loci of SNPs with significant GWAS results for reproductive 217 traits.

218 SLAF-seq is a simplified deep genome-sequencing technique, which can be used to obtain and 219 accurately type numerous molecular markers using bioinformatics. This technology has been 220 successfully implemented in studying many species such as cotton (Li et al., 2017) (IF: 7.44) and 221 soybean (Han et al., 2016) (IF: 7.33). In this study, 1192168 SLAF tags from the sheep reference genome were predicted using the Hpy166II + EcoRV-HF® enzyme combination. The average sequencing depth was 13.06x, and 685,300 SNPs were found. Therefore, the SLAF-seq method used in this experiment provided comprehensive genomic variation information. Considering the enormous differences in genome sequences between European and Chinese sheep breeds, these experimental data show that SLAF-seq is a powerful method with considerable potential for use in studying more breeds. Therefore, the SLAF-seq method could be considered a highly efficient option for sheep genome research.

Molecular markers and candidate genes of litter size in sheep

Genetic markers for the number of lambs in sheep have been studied, but the reports are limited. More studies have focused on the ovulation rate than on the litter size. Current studies have shown that GDF9, BMP15, and BMPR-IB genes and 13 mutation loci $(F e c B B, F e c X B$, $F e c X G, F e c X G R$, FecXH, FecXI, FecXL, FecXO, FecXR, FecGH, FecGT, and V371M) (Mullen \& Hanrahan, 2014) are the major genes affecting litter size or ovulation rate in sheep. However, these markers are not stable in other sheep breeds and are not the major genes affecting their litter size, indicating that there are other genes responsible for litter size and interspecies 
238 resources and the Hetian sheep breeds used in this experiment, which often produce many lambs,

239 have rarely been used an experimental model.

240 In this study, 30 loci of seven ovulation-related genes (FSHR, COIL, GUCY1A1, CXCL12,

241 SLK, LOC101107541, and LOC101107119) were selected for KASP validation. Results showed

242 that nine out of the 30 loci of three genes (CXCL12, FSHR, and COIL) were successfully

243 genotyped. The other four genes are rarely reported in livestock and have mostly been studied in

244 humans and mice. Among them, the SLK gene affects the development of the ovary and follicle

245 (An, 2012), CXCL12 affects early development of the placenta and placental membrane (Quinn

246 et al., 2016; Sanchez et al., 2017), and LOC101107541 and LOC101107119 participate in the

247 oxytocin signalling pathway and indirectly regulate the production of ovarian steroids. The

248 results need to be further verified.

\section{Relationship between $F S H R$ gene and sheep reproduction}

250

This study concluded that the FSHR gene may be one of the important genes affecting litter

251 size of Hetian sheep. Follicular stimulating hormone receptor (FSHR) is a member of the glycoprotein superfamily of $\mathrm{G}$ protein-coupled receptors and plays an important role in follicular

253 development in animals. FSHR is mainly expressed in granulosa cells of follicles in super ovulated and normal lambs, and there are positive signals in primordial follicles. No positive signals are observed in primordial follicles in normal adult sheep, and the expression of FSHR is decreased in large dominant follicles (Chu et al., 2012). Some studies have reported the fulllength coding sequences of six FSHR spliced forms in sheep. The open reading frames are 695aa, 694aa, 648aa, 633aa, 595aa, and 533aa. While 533aa is not found in the ovaries of lambs, 694aa and 648aa are not found in adult sheep (Jiang, 2014). In this study, FSHR was found to be associated with follicular development.

Chu et al. (2012) detected 50 SNPs in the regulatory region of the FSHR gene in two highreproductive (small-tailed $\mathrm{Han}$ and $\mathrm{Hu}$ ) and two low-reproductive (Kaolidai and Chinese Merino) sheep breeds. Four mutations in the sheep FSHR gene were detected using polymerase chain reaction-single-strand conformation polymorphism (PCR-SSCP) technology, suggesting that the FSHR gene may significantly affect the litter size of sheep (Chu et al, 2012). Pan et al. (2014) cloned 50 flanking regions of the sheep FSHR gene and analysed its genomic structure. be significantly correlated $(\mathrm{P}<0.1)$ with litter size (Pan et al., 2014). Some of the present observations are consistent with those of previous studies. 
272 three genotypes, namely GG, GA, and AA in the Xinjiang Hetian and Bashbai sheep breeds.

273 Correlation analysis comparing the different genotypes and litter size showed that the GG

274 genotype was highly correlated with litter size, which further verified the reliability of SLAF-seq

275 and verified that FSHR is a candidate gene affecting the litter size in sheep.

276 Relationship between Coilin (COIL) gene and sheep reproduction

277

278

Coilin is a characteristic protein of the Cajal body, which is a conserved nuclear organelle that participates in many aspects of small ribonucleoprotein (RNP) biogenesis (Gall et al., 1999).

279 Coilin is necessary for Cajal body formation and recruitment of spliced nucleoproteins (snRNPs) to modify protein complexes that guide RNA and motor neurons (SMN). When coilin is deleted, the remaining Cajal bodies lose contact with SMN complexes. Because SMN is considered a necessary condition for snRNPs assembly and internal circulation, the lack of interaction between SMN and coilin in the nucleus may lead to a decline in RNP assembly capacity, which may have down-stream effects on development and gametes, thus, affecting litter size. Walker et al. (2009) studied the effect of coilin removal on the overall viability and reproductive success of mice. The results showed that the number of oocytes that could fertilize was significantly reduced after COIL knockout, and the number of litters per foetus was low, showing obvious fertility and reproductive deficiencies (Walker et al., 2009). COIL was found to be closely related to litter size. In this study, the association between genotype and litter size revealed that the CC genotype was significantly associated with litter size, and we verified that COIL is one of the candidate genes affecting the litter size in sheep.

\section{Relationship between $G U C Y 1 A 1$ gene and sheep reproduction}

Guanylate cyclase (GC) is an enzyme that converts guanylic-5'-triphosphate into cycloguangan-3',5'-monophosphate (Pyriochou \& Papapetropoulos, 2005). As a membranebinding molecule, GC exists in the membrane-bound and cytoplasmic forms. Soluble GC (sGCX), an isodimer enzyme composed of alpha and beta subunits, is the only receptor for nitric oxide (NO) as a biological messenger identified to date and is closely involved in various signal transduction pathways (Peter et al., 2010). The sGCX collector (GUCY1A1) was found to be involved in hormone regulation, oxytocin signalling pathway, and reproductive capacity. In this study, we found that the mutation site of the GUCY1A1 gene (g. 43266624C $>\mathrm{T}$ ) was significantly correlated with litter size of Hetian sheep, thus, verifying the correctness of the sequencing results.

The results of KASP typing showed that three genes (FSHR, GUCY1A1, and COIL) were successfully typed. The genotypes of mutated loci correlated with high fecundity and average litter size of field sheep. The results showed that the genotypes of FSHR (g.75320741G $>$ A) were 
306 extremely significantly correlated with high fecundity and average litter size of field sheep.

307 GUCY1A1 (g.43266624C > T) and COIL (g.7321466C > G) genes were significantly correlated

308 with high fecundity and average litter size of field sheep. The results of this study confirm that

309 mutations of these three genes caused changes in litter size of sheep and validated the SLAF

310 sequencing technology.

311 Molecular marker screening is a major step in molecular breeding. SNP markers are third-

312 generation molecular markers and are currently the mainstream molecular markers. KASP

313 genotypes SNPs and indels at specific sites for precise genetic determination. Compared to other

314 detection methods, KASP has higher throughput, is faster, more cost-effective, and produces

315 more accurate results.

316 Breeding sheep with high fertility is important for mutton production. Molecular marker

317 screening is used to improve early molecular selection. In this study, the KASP technology was

318 used to verify three sites with significant or extremely significant effects on litter size, which

319 could lead to breakthroughs in the development of sheep multi-lamb genes and markers, and

320 improvement of the efficiency of molecular breeding of sheep with high fecundity. It can be used

321 to improve other low-community groups and the efficiency of meat production in sheep. The

322 study showed that SLAF-seq and KASP are cost-effective tools for selecting the desirable

323 genotypes in sheep breeding programs.

324

325 Conclusions

326 In this study, DNA of Chinese indigenous sheep from southern Xinjiang with different litter

327 size fecundity were sequenced using the SLAF-seq technique. GWAS of SNPs that might cause

328 differences in litter size was carried out. In total, 155 genes with significant mutations were

329 obtained using SNP loci, gene annotation, and pathway analysis, of which 17 genes were related

330 to reproductive traits. Seven candidate genes closely related to litter size and ovulation were

331 identified through literature search and comprehensive analysis. The KASP technique was used

332 to verify the role of the seven genes. Among the seven selected genes, only FSHR (GenBank ID:

333 443299, g. 75320741G>A site), GUCY1A1 (GenBank ID: 101110000, g. 43266624C >T site),

334 and COIL (GenID: 101123134, g. 7321466C > G site) loci were identified to be significant among

335 different genotypes as molecular markers to detect the differences in litter size of sheep

336 population from Xinjiang. This finding provides new information that explains the regulatory

337 mechanism underlying sheep fecundity and identifies molecular markers for litter size traits. The

338 sub-markers are of great significance for breeding of high-fecundity sheep breeds.

339 


\section{Acknowledgements}

341 This research was supported by NSFC (No. U1603232), National Key R\&D Program of China

342 (No. 2018YFD0502100), Autonomous Region Key Research and Development Project

343 (No.2017B01005-2-1), and Xinjiang Agricultural University Graduate Research and Innovation

344 Project (No.XJAUGRI2017033).

345 We would like to thank all the participants of the study. We would also like to thank Editage

346 [www.editage.cn] for English language editing.

347

348

\section{References}

Alexander DH, Novembre J, Lange K. 2009. Fast model-based estimation of ancestry in unrelated individuals. Genome Research 19:1655-1664 DOI: 10.1101/gr.094052.109.

An N. 2012. Localization dynamics and preliminary research Function of Ste20-like kinase(SLK) during Mouse Oocytes maturation. Jilin Agricultural University.

Bradbury P, Zhang Z, Kroon D, Casstevens T, Y, and Buckler E. 2007. TASSEL: software for association mapping of complex traits in diverse samples. Bioinformatics 23:2633-2635 DOI: $10.1093 /$ bioinformatics/btm308.

Davey JW, Cezard T, Fuentes - Utrilla P, Eland C, Gharbi K, Blaxter ML. 2013. Special features of RAD Sequencing data: implications for genotyping. Molecular ecology 22: 3151-3164 DOI: $10.1111 / \mathrm{mec} .12084$

Deniskova T, Dotsev AV, Selionova M, Wimmers K, Reyer H, Kharzinova VR. 2017. Wholegenome single nucleotide polymorphism study of Romanov sheep. J Anim Sci 95:339340 DOI:10.2527/asasann.2017.696.

Eiriksson JH, Sigurdsson A. 2017. Sources of bias, genetic trend and changes in genetic correlation in carcass and ultrasound traits in the Icelandic sheep population. Iceland Agric Sci 30:3-12 DOI: 10.16886/IAS.2017.01.

Gall JG, Bellini M, Wu Z, and Murphy C. 1999. Assembly of the nuclear transcription and processing machinery: Cajal bodies (coiled bodies) and transcriptosomes. Molecular Biology of the Cell 10:4385 DOI: 10.1091/mbc.10.12.4385. 
372 Hardy OJ, Vekemans X. 2002. SPAGeDI: A versatile computer program to analyse spatial

373

374

375

376

377

378

379

380

381

382

383

384

385

386

387

388

389

390

391

392

393

394

395

396

397

398

399

400

401

402

403 genetic structure at the individual or population levels. Molecular Ecology Notes 2(4):618-620 DOI: 10.1046/j.1471-8286.2002.00305.x

Han Y, Zhao X, Liu D, Li Y, Lightfoot DA, Yang Z, Zhao L, Zhou G, Wang Z, and Huang L. 2016. Domestication footprints anchor genomic regions of agronomic importance in soybeans. New Phytologist 209:871-884 DOI: 10.1111/nph.13626

Hanrahan JP, Gregan SM, Philippe M, Michael M, Davis GH, Richard P, and Galloway SM. 2004. Mutations in the genes for oocyte-derived growth factors GDF9 and BMP15 are associated with both increased ovulation rate and sterility in Cambridge and Belclare sheep (Ovis aries). Biology of Reproduction 70:900-909 DOI: 10.1095/biolreprod.103.023093.

He JN, Zhang BY, Chu MX, Feng T, Cao GL, Di R, Fang L, Huang DW, Tang QQ, and Li N. 2012. Polymorphism of insulin-like growth factor 1 gene and its association with litter size in Small Tail Han sheep. Molecular Biology Reports 39:9801-9807 DOI: 10.1007/s11033-012-1846-y.

Jiang WD, Zhang J, Song XM, Ai DX, Li LR, and Shi CQ. 2017. Extraction of total RNA from the overy of oira black sheep. Heilongjiang Animal Science and Veterinary Medicine:6263.

Jiang XJ. 2014. Expression analysis of AMH、FSHR、LHR in ovine ovarian follicles, Shihezi University.

Köchl S, Niederstätter H, and Parson W. 2005. DNA Extraction and Quantitation of Forensic Samples Using the Phenol-Chloroform Method and Real-Time PCR. Methods in Molecular Biology 297:13 DOI: 10.1385/1-59259-867-6:013.

Kozich JJ, Westcott SL, Baxter NT, Highlander SK, and Schloss PD. 2013. Development of a dual-index sequencing strategy and curation pipeline for analyzing amplicon sequence data on the MiSeq Illumina sequencing platform. Applenvironmicrobiol 79:5112-5120 DOI: 10.1128/AEM.01043-13.

Li H, and Durbin R. 2009. Fast and accurate short read alignment with Burrows-Wheeler transform. Bioinformatics. 25:1754-1760 DOI: 10.1093/bioinformatics/btp324.

Li H, Handsaker B, Wysoker A, Fennell T, and Ruan J. 2009a. The Sequence Alignment-Map format and SAMtools. Bioinformatics 25:2078-2079 DOI: 10.1093/bioinformatics/btp352. 
404 Li R, Yu C, Li Y, Lam TW, Yiu SM, Kristiansen K, and Wang J. 2009b. SOAP2: an improved

405

406

407

408

409

410

411

412

413

414

415

416

417

418

419

420

421

422

423

424

425

426

427

428

429

430

431

432

433

434

435 ultrafast tool for short read alignment. Bioinformatics 25:1966-1967 DOI: 10.1093/bioinformatics/btp336.

Li T, Ma X, Li N, Zhou L, Liu Z, Han H, Gui Y, Bao Y, Chen J, and Dai X. 2017. Genome-wide association study discovered candidate genes of Verticillium wilt resistance in upland cotton (Gossypium hirsutum L.). Plant Biotechnology Journal 15:1520-1532 DOI: 10.1111/pbi.12734.

Mckenna A, Hanna ME, Sivachenko A, Cibulskis K, Kernytsky A, Garimella K, Altshuler D, Gabriel S, Daly M, and Depristo MA. 2010. The Genome Analysis Toolkit: a MapReduce framework for analyzing next-generation DNA sequencing data. Genome Research 20:1297-1303 DOI: 10.1101/gr.107524.110.20.

Mullen MP, and Hanrahan JP. 2014. Direct Evidence on the Contribution of a Missense Mutation in GDF9 to Variation in Ovulation Rate of Finnsheep. Plos One 9:e95251 DOI: 10.1371/journal.pone.0095251.

Pan X, Liu S, Li F, Wang W, Li C, Ma Y, and Li T. 2014. Molecular characterization, expression profiles of the ovine FSHR gene and its association with litter size. Molecular Biology Reports 41:7749-7754 DOI: 10.1007/s11033-014-3666-8.

Peter O, Shruti S, Albert G, Anthony A, Cynthia H, Johengen MJ, Jong-Hau H, Sohrab F, Black SM, and Fineman JR. 2010. Alterations in cGMP, soluble guanylate cyclase, phosphodiesterase 5, and B-type natriuretic peptide induced by chronic increased pulmonary blood flow in lambs. Pediatric Pulmonology 42:1057-1071 DOI: 10.1002/ppul.20696.

Purcell S, Neale B, Todd-Brown K, Thomas L, Ferreira MA.R, Bendr D, Maller J, Sklar P, De Bakker P, Daly M, Sham P. 2007. PLINK: A Tool Set for Whole-Genome Association and Population-Based Linkage Analyses. American Journal of Human Genetics 81(3):0575 DOI: $10.1086 / 519795$.

Pyriochou, and Papapetropoulos. 2005. Soluble guanylyl cyclase: more secrets revealed. Cellular Signalling 17:407-413 DOI: 10.1016/j.cellsig.2004.09.008.

Quinn KE, Reynolds LP, Grazul-Bilska AT, Borowicz PP, and Ashley RL. 2016. Placental development during early pregnancy: Effects of embryo origin on expression of chemokine ligand twelve (CXCL12). Placenta 43:77-80 DOI: 10.1016/j.placenta.2016.05.008. 
436 Sanchez NS, Quinn KE, Ashley AK, and Ashley RL. 2017. In the ovine pituitary, CXCR4 is

437

438

439

440

441

442

443

444

445

446

447

448

449

450

$$
\text { localized in gonadotropes and somatotropes and increases with elevated serum }
$$
progesterone. Domest Anim Endocrinol 62:88-97.

Walker MP, Liping T, and A Gregory M. 2009. Reduced viability, fertility and fecundity in mice lacking the cajal body marker protein, coilin. Plos One 62:88-97 DOI: 10.1016/j.domaniend.2017.10.003.

Wang S, Li XL, Niu ZG, and Shi HC. 2017. Whole genomic association analysis of polyembryonic gene in Duolong sheep. Jiangxi Agricultural Journal 29:77-81.

Wickham, H., 2015. ggplot2. Wiley Interdisciplinary Reviews Computational Statistics 3(2),180185.

Yue GH. 1996. Reproductive characteristics of Chinese Hu sheep. Animal Reproduction Science 44:220-230 DOI:10.1016/0378-4320(96)01562-X.

Zhiwu Z, Elhan E, Chao-Qiang L, Todhunter RJ, Tiwari HK, Gore MA, Bradbury PJ, Jianming Y, Arnett DK, and Ordovas JM. 2010. Mixed linear model approach adapted for genomewide association studies. Nature Genetics 42:355-360 DOI: 10.1038/ng.546. 
Table $\mathbf{1}$ (on next page)

Information of Four Genes 
Table.1 Information of Four Genes

\begin{tabular}{|c|c|c|}
\hline Gene & Position & Primer Sequence \\
\hline \multirow[t]{3}{*}{ GUCY1A1 } & 43266624 & F1: GAAGGTCGGAGTCAACGGATTGGAGTGGGCCAGCAGCTAC \\
\hline & & F2: GAAGGTGACCAAGTTCATGCTGGAGTGGGCCAGCAGCTAT \\
\hline & & R1: GTTCTTGTCAGGGACACCTGG \\
\hline \multirow[t]{3}{*}{$S L K$} & 23608558 & F1:GAAGGTCGGAGTCAACGGATTCTTGCGAGATGAAGCCAAGC \\
\hline & & F2:GAAGGTGACCAAGTTCATGCTCTTGCGAGATGAAGCCAAGT \\
\hline & & R1: ACATTCTGAAATTTGGACAGCTC \\
\hline \multirow[t]{3}{*}{ COIL } & 7314134 & F1: GAAGGTCGGAGTCAACGGATTCCATGAAAGAACCTGGGAAA \\
\hline & & F2: GAAGGTGACCAAGTTCATGCTCCATGAAAGAACCTGGGAAC \\
\hline & & R1: CCTCAGCTCCATTTTCGTTG \\
\hline \multirow[t]{3}{*}{ COIL } & 7321466 & F1: GAAGGTCGGAGTCAACGGATTGACTCCGAGGAGGAATCGC \\
\hline & & F2: GAAGGTGACCAAGTTCATGCTGACTCCGAGGAGGAATCGG \\
\hline & & R1: GTGGCATGGTCGTCCGTAC \\
\hline \multirow[t]{4}{*}{ COIL } & 7321563 & F1:GAAGGTCGGAGTCAACGGATTGCACAGTCTGTGAAAGAGTGGA \\
\hline & & F2: \\
\hline & & GAAGGTGACCAAGTTCATGCTGCACAGTCTGTGAAAGAGTGGG \\
\hline & & R1: TCTAGCAGGAAGAGCTTTAGGG \\
\hline \multirow[t]{3}{*}{ FSHR } & 75132817 & F1: GAAGGTCGGAGTCAACGGATTAGCCCAAGCTCAGGAATGC \\
\hline & & F2: GAAGGTGACCAAGTTCATGCTGAGCCCAAGCTCAGGAATGT \\
\hline & & R1: GGTGGATGGATAAGTAAACATGG \\
\hline \multirow[t]{4}{*}{ FSHR } & 75320579 & F1: \\
\hline & & GAAGGTCGGAGTCAACGGATTGGACAGGGAAGACTCACTCACA \\
\hline & & F2: GAAGGTGACCAAGTTCATGCTGACAGGGAAGACTCACTCACG \\
\hline & & R1: CTCACCTACCCCAGCCACT \\
\hline \multirow[t]{4}{*}{ FSHR } & 75320741 & F1: \\
\hline & & GAAGGTCGGAGTCAACGGATTGATATTTCAAGAACCAGGATCCA \\
\hline & & F2: GAAGGTGACCAAGTTCATGCTATATTTCAAGAACCAGGATCCG \\
\hline & & R1: CAGCTTCTTAAGATTTTCTAAGCC \\
\hline \multirow[t]{3}{*}{ FSHR } & 75132820 & F1: GAAGGTCGGAGTCAACGGATTATGATGCTGGCAGCATGGT \\
\hline & & F2: GAAGGTGACCAAGTTCATGCTATGATGCTGGCAGCATGGC \\
\hline & & R1: CATCACCCACGCCATGCAG \\
\hline
\end{tabular}


Figure 1

Principal component analysis (PCA) of Hetian(Mulitiple) and Bashbay(Single)sheep breeds

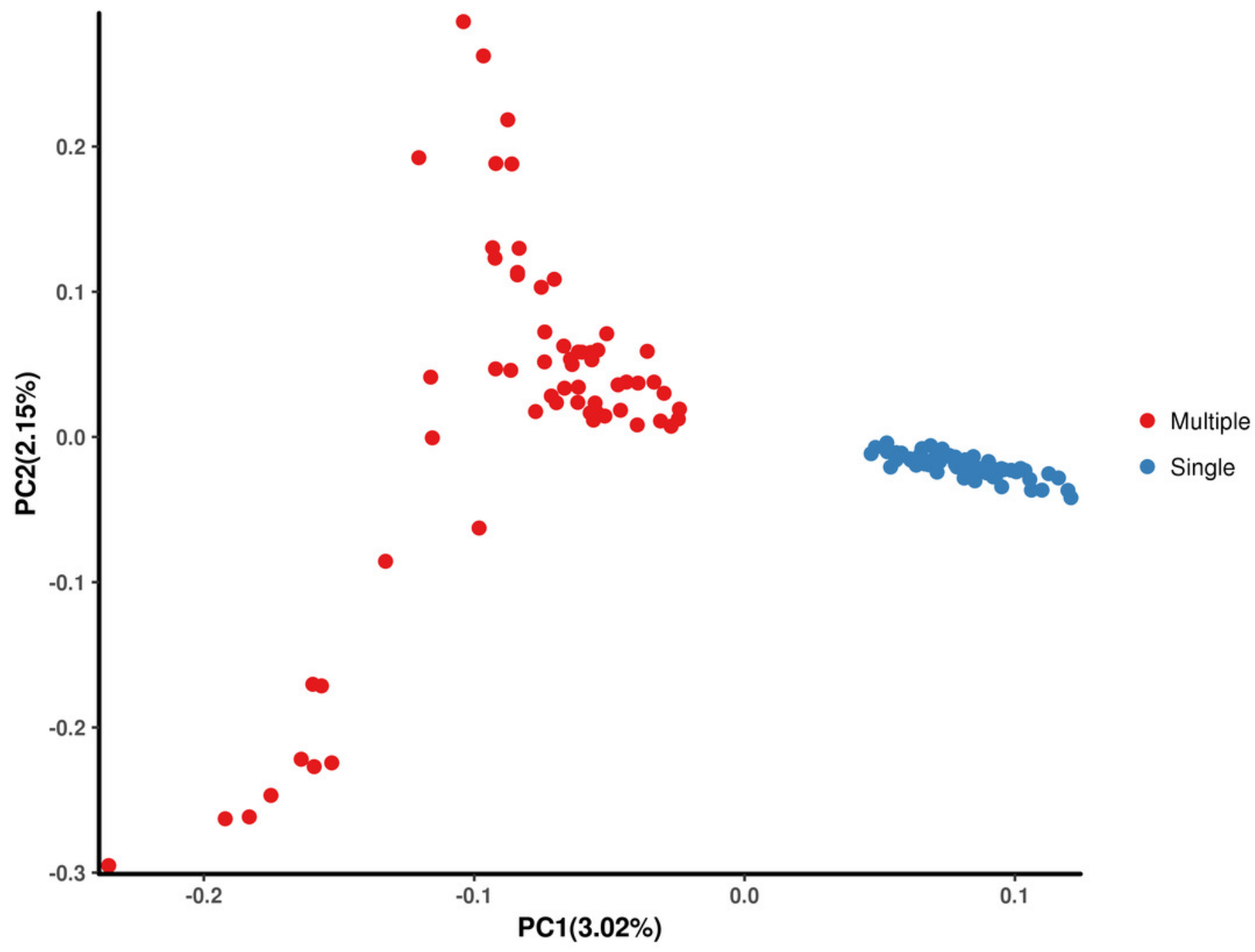


Figure 2

Manhattan plot for genome-wide association study on GLM model

Note: the scale on the X-axis represents ID of chromosomes, NC_019458.2-

NC_019483.2represents ID 1-26 of chromosomes, $X$ chromosome is represented by NC_019484.2. The scale on the Y-axis is the $-\log 10$ (P-value)score of association analysis.

The rad dashed line indicates genome-wide significance of suggestive association.

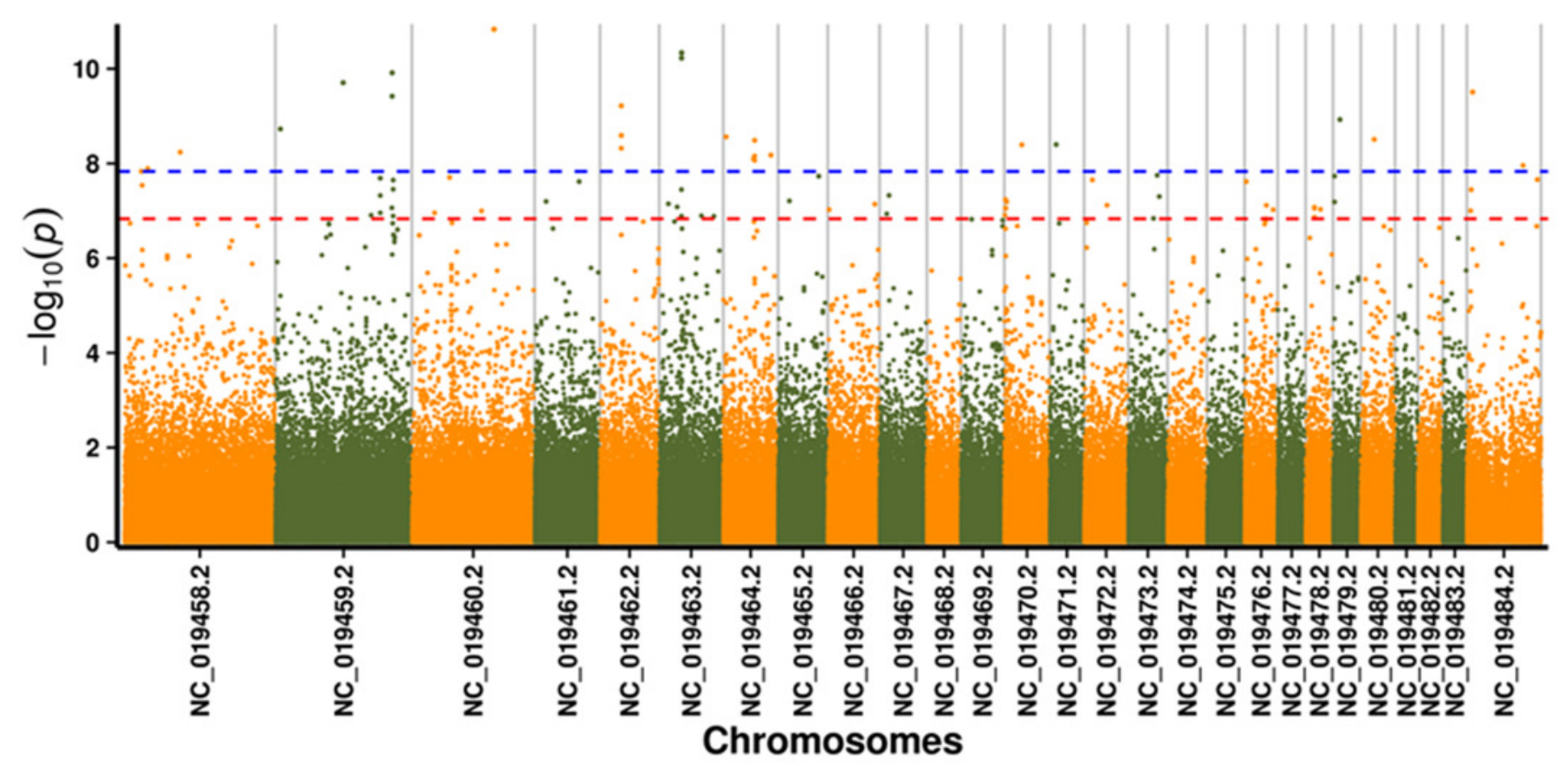


Figure 3

Manhattan plot for genome-wide association study on MLM model

Note: the scale on the X-axis represents ID of chromosomes, NC_019458.2-

NC_019483.2represents ID 1-26 of chromosomes, $X$ chromosome is represented by NC_019484.2. The scale on the Y-axis is the $-\log 10$ (P-value)score of association analysis.

The rad dashed line indicates genome-wide significance of suggestive association.

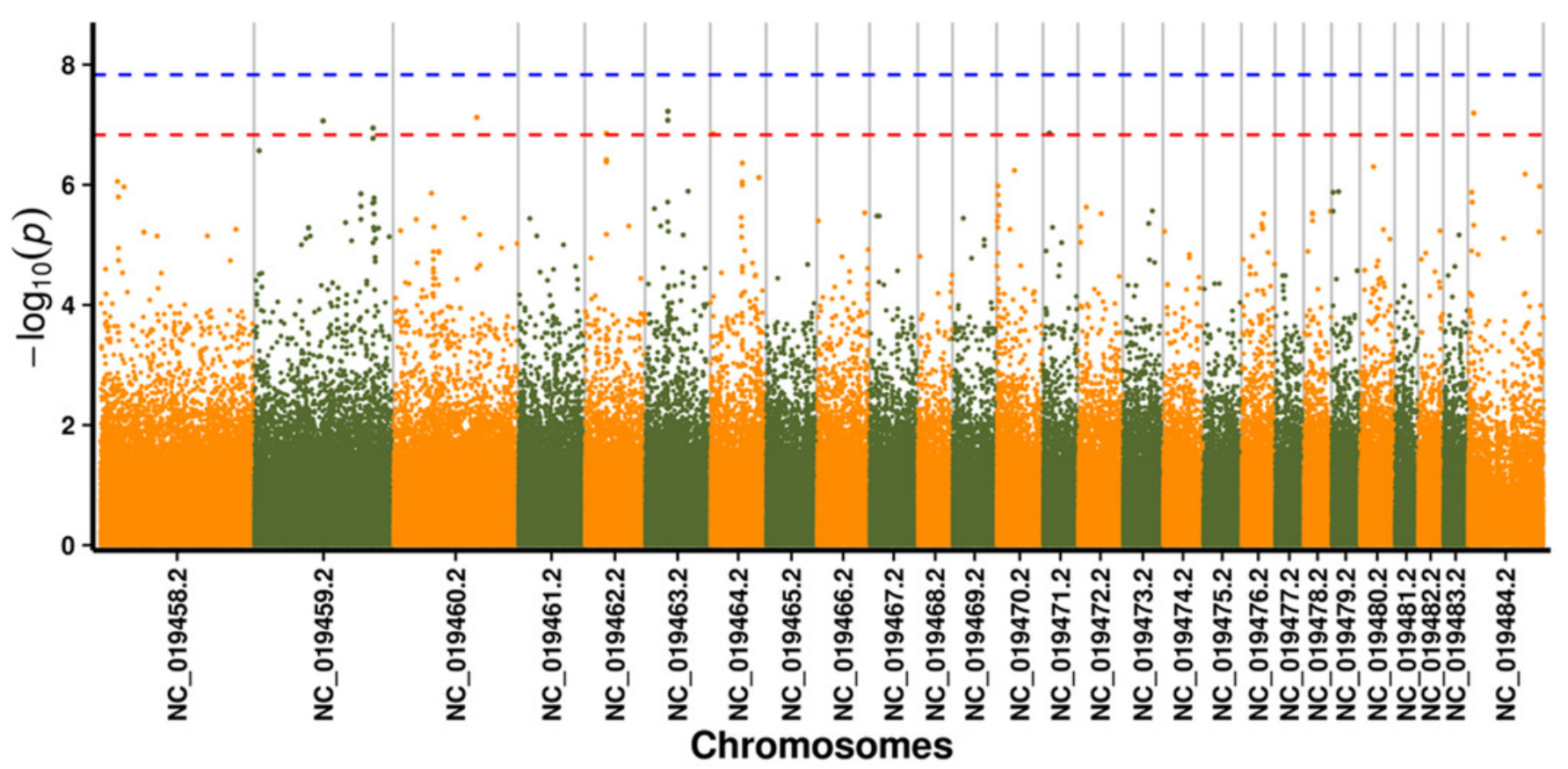


Figure 4

Venn diagram of two methods

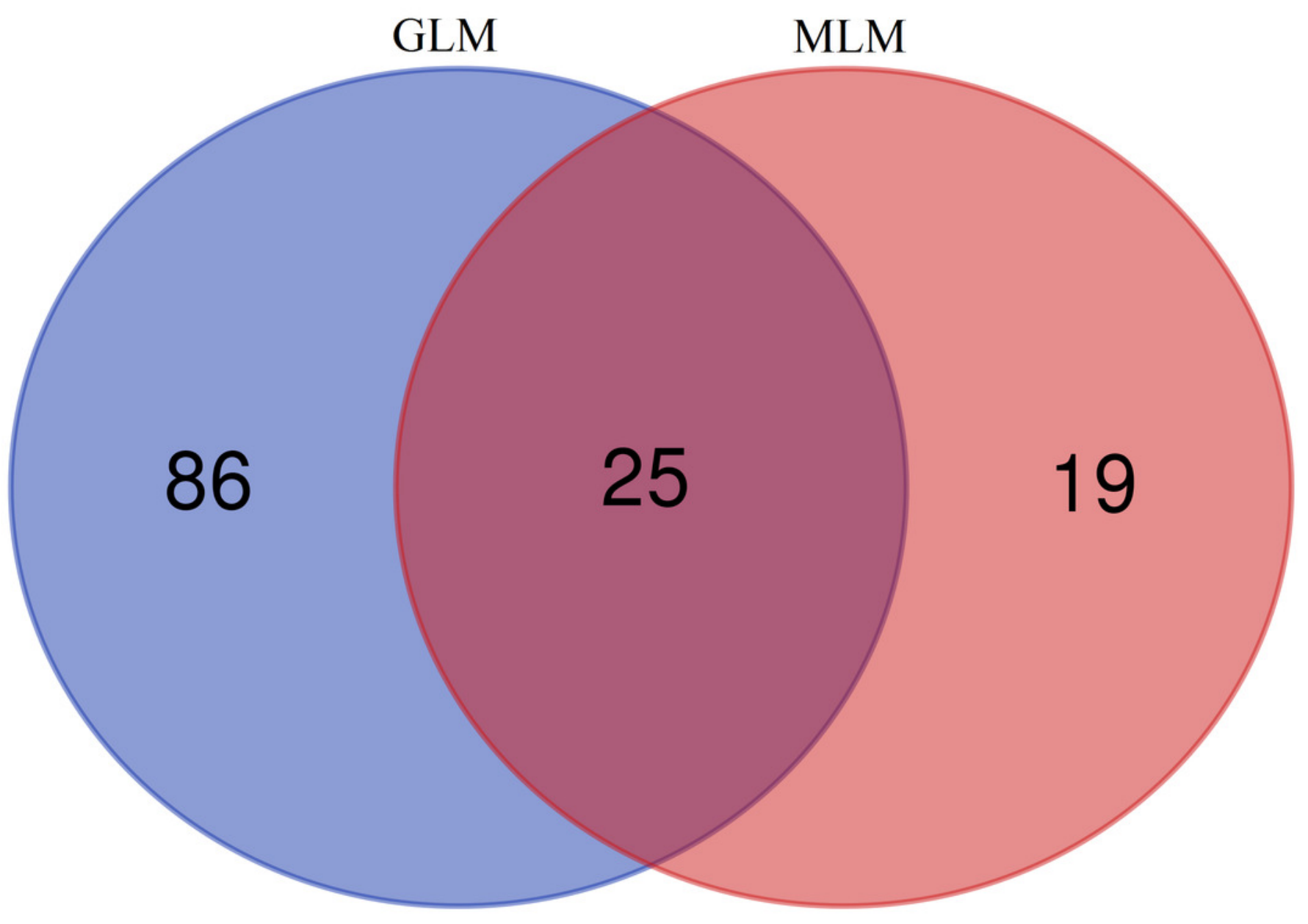


Figure 5

The results of quantile-quantile (Q-Q) plot for Litter size trait in GLM model

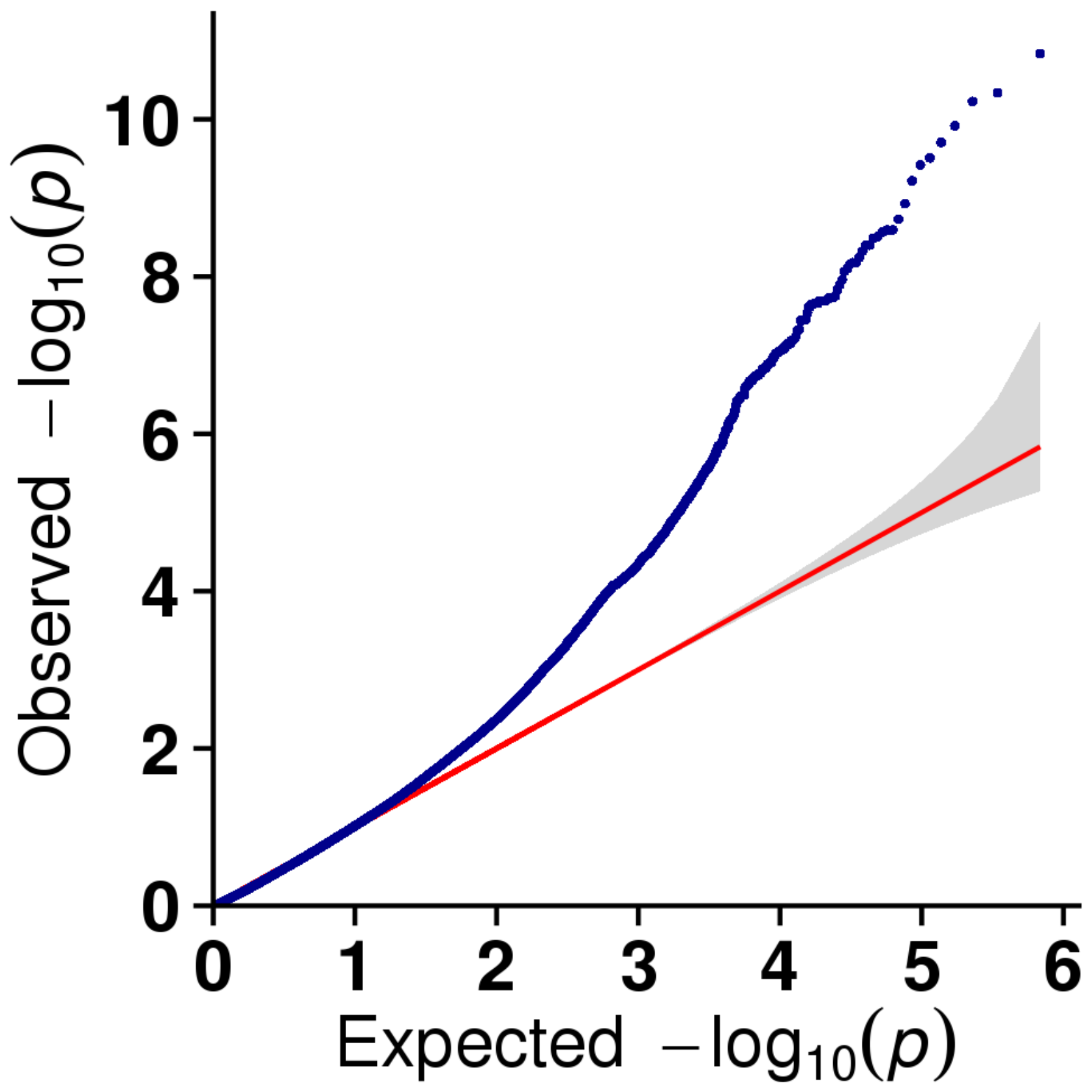


Figure 6

The results of quantile-quantile (Q-Q) plot for Litter size trait in MLM model

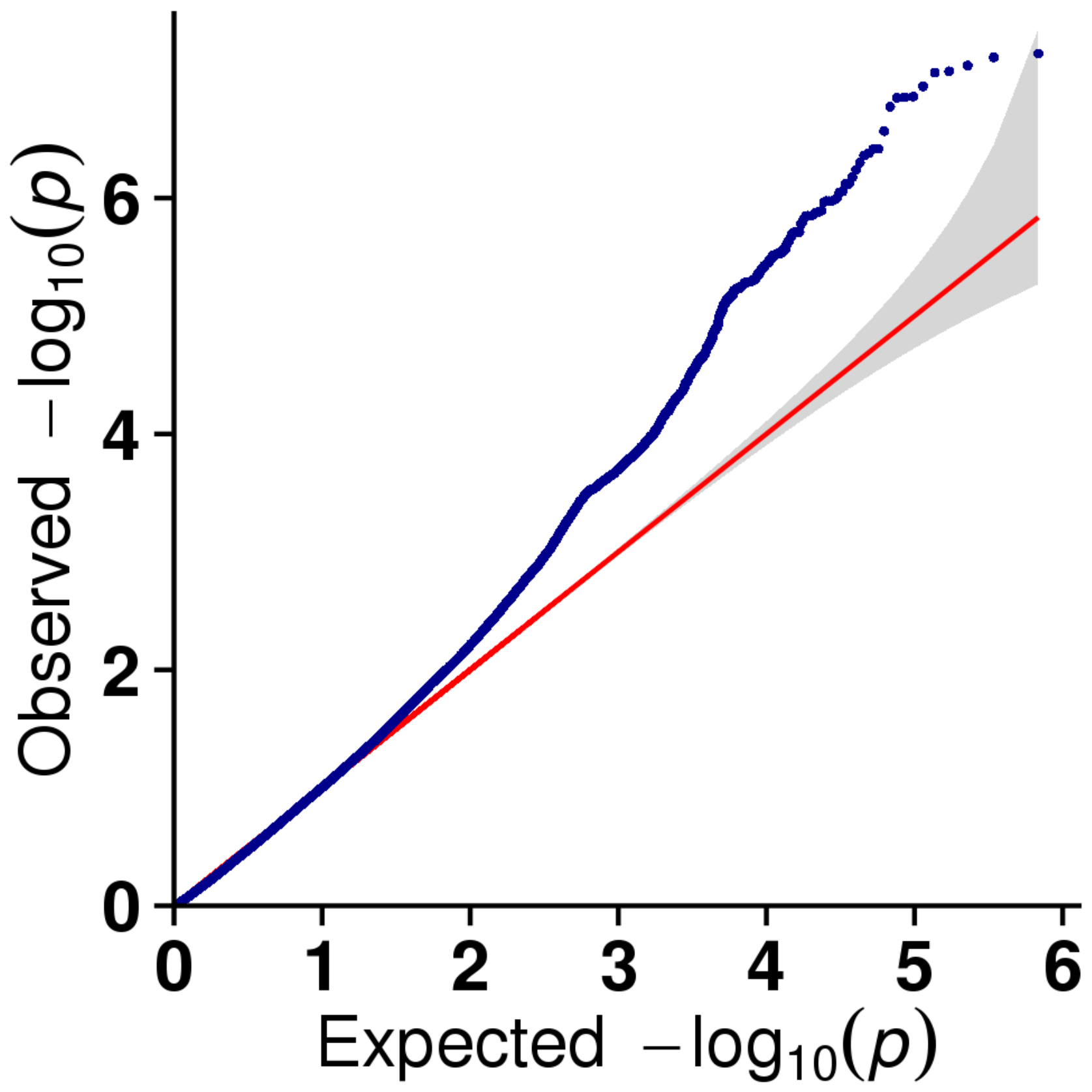


Figure 7

Genotyping results of GUCY1A1 gene

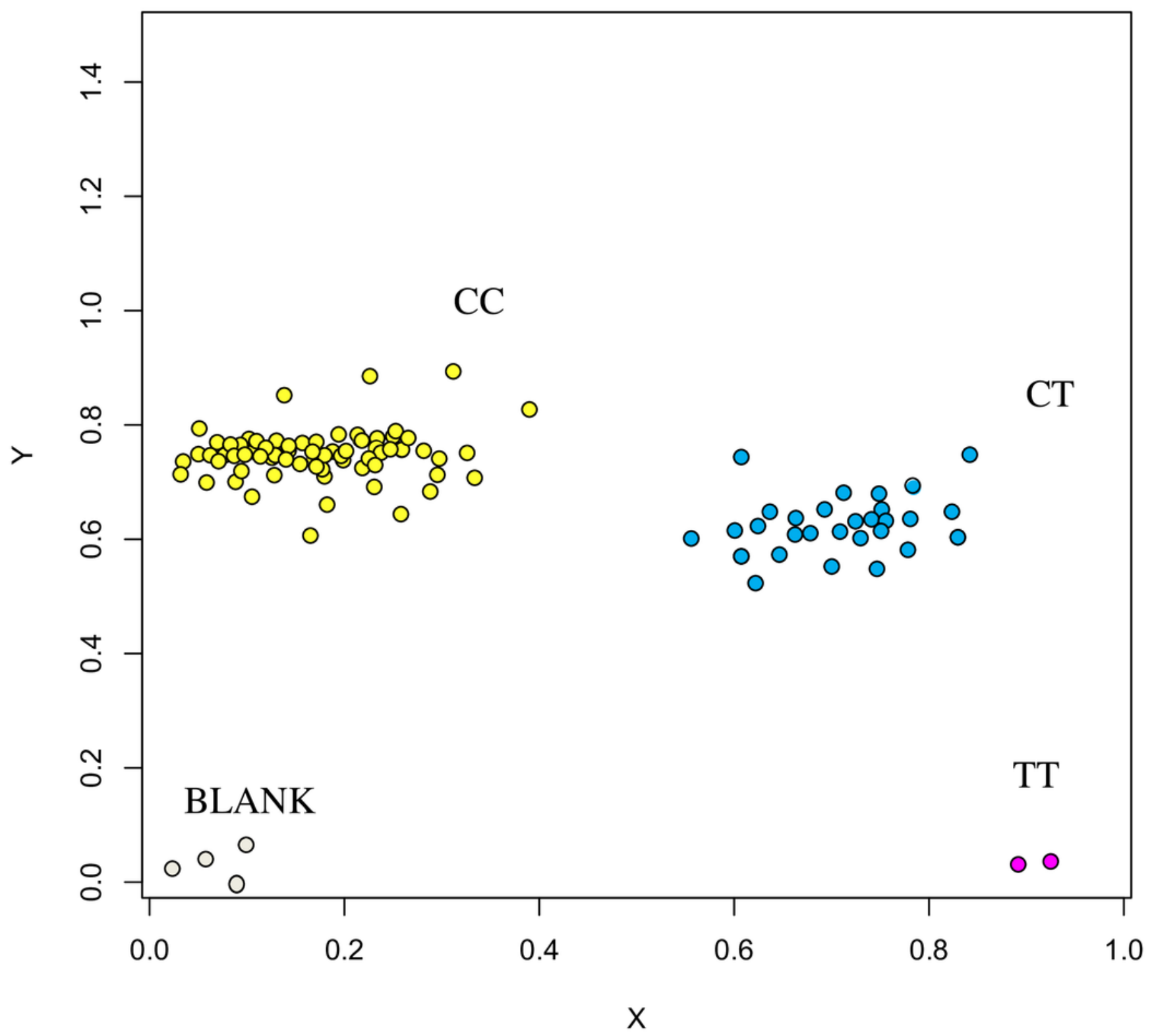


Figure 8

Genotyping results of FSHR gene

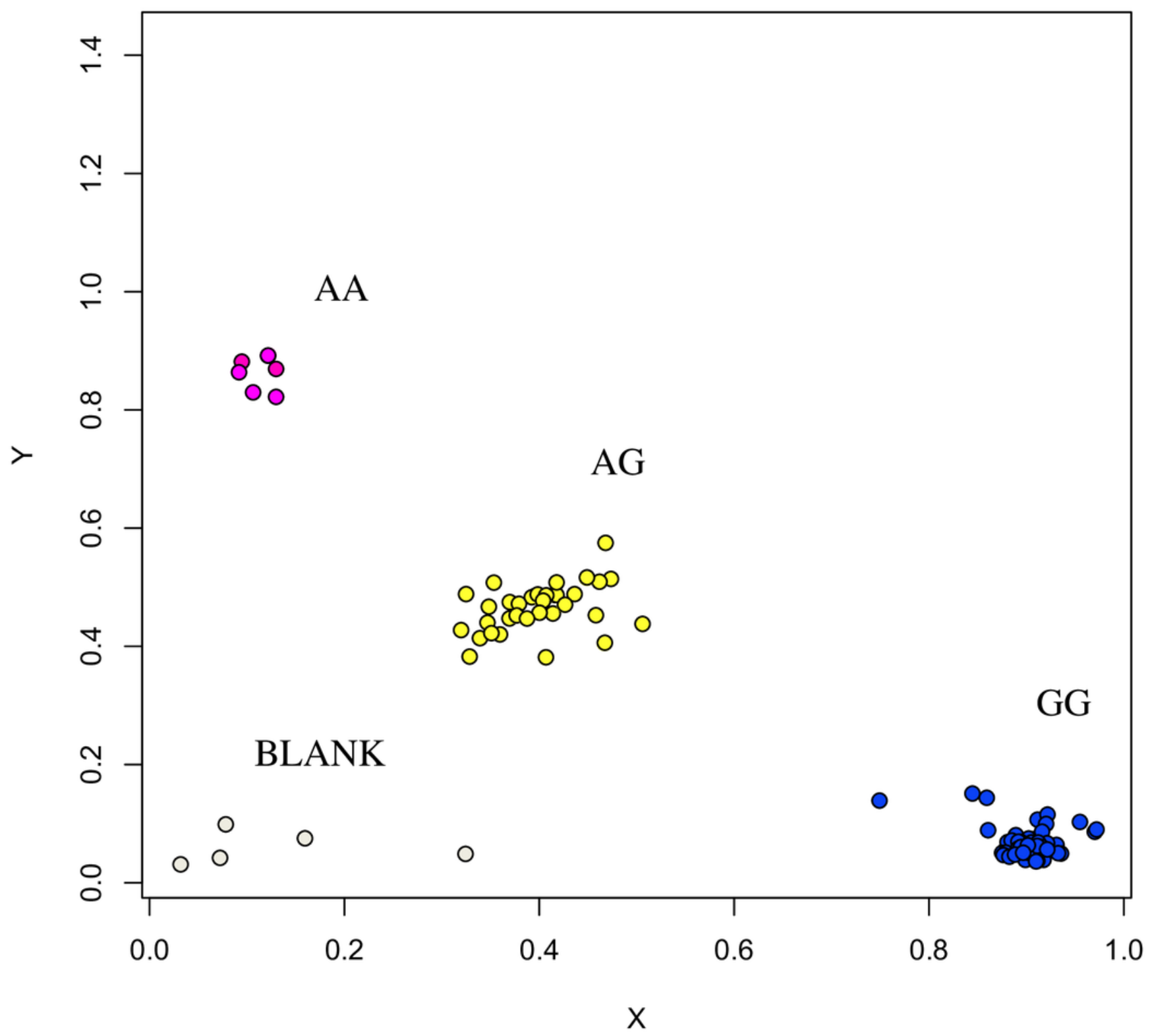


Figure 9

Genotyping results of COIL gene

SNP125_2

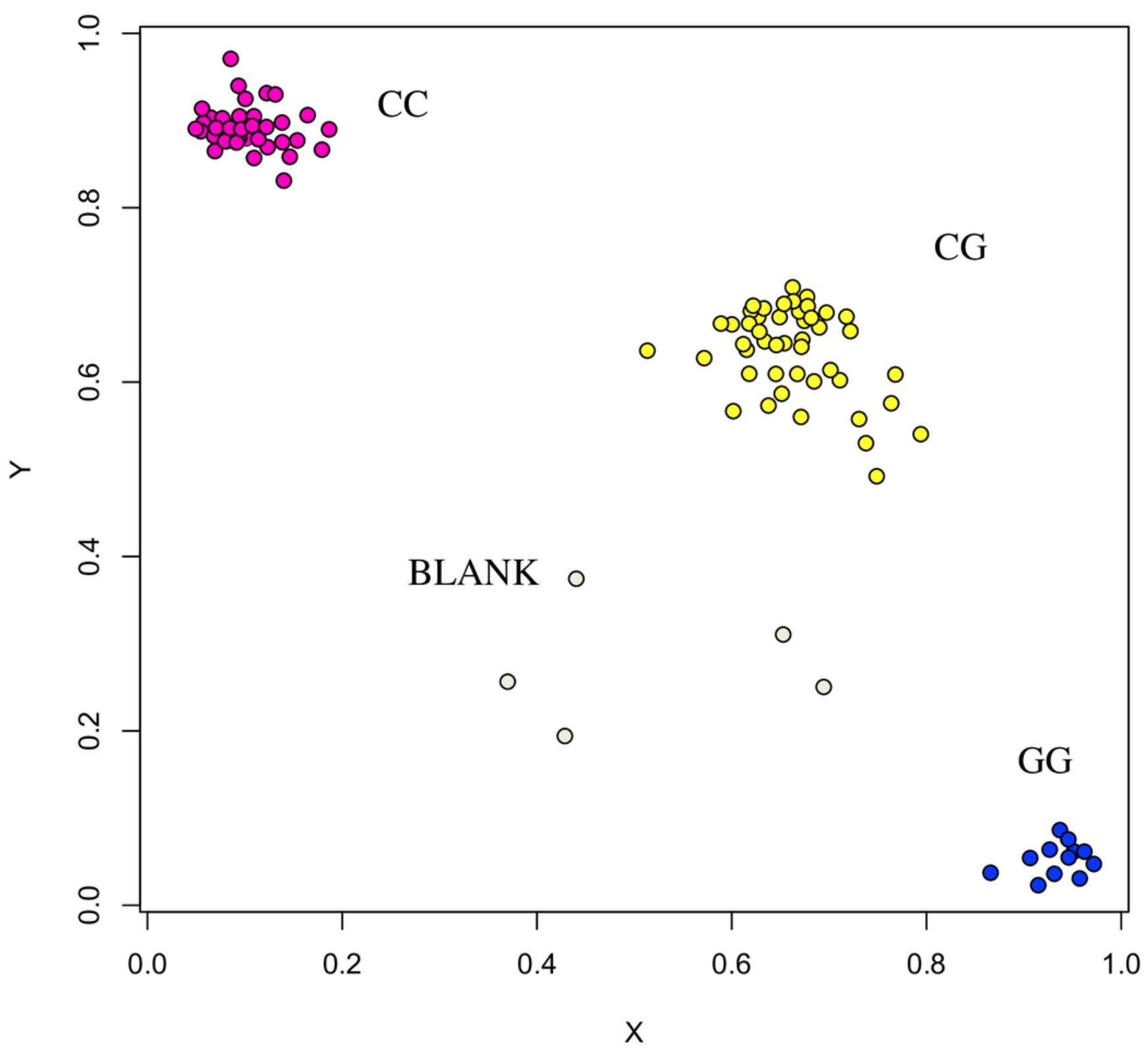


Figure 10

Correlation analysis between different genes and litter size

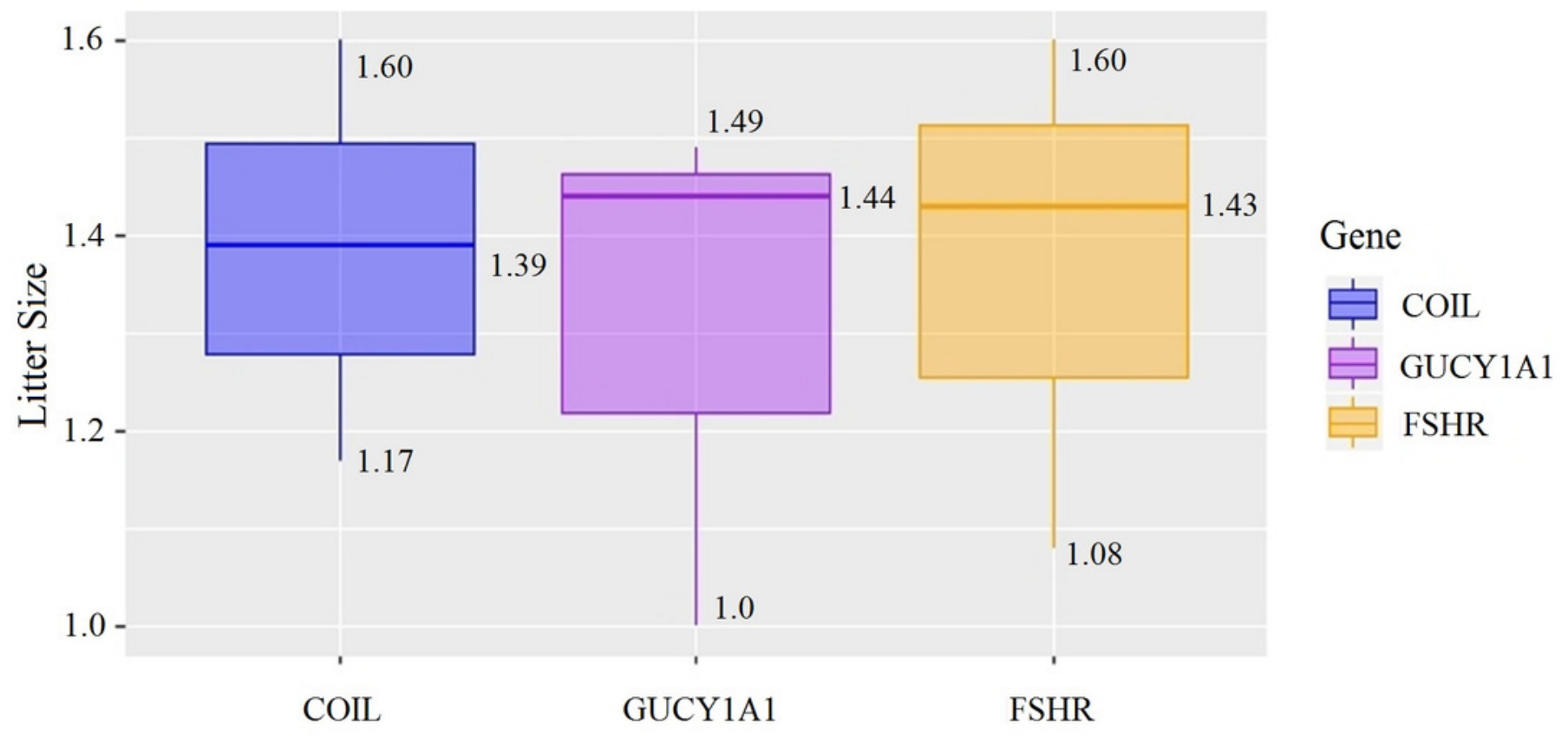

